Journal of Computational Electronics 3: 25-31, 2004 (c) 2004 Kluwer Academic Publishers. Manufactured in The Netherlands.

\title{
Electrodiffusion Model Simulation of Ionic Channels: 1D Simulations
}

\author{
CARL L. GARDNER* \\ Department of Mathematics and Statistics, Arizona State University, Tempe, AZ 85287, USA \\ gardner@math.asu.edu \\ WOLFGANG NONNER ${ }^{\dagger}$ \\ Department of Physiology and Biophysics, University of Miami Medical Center, Miami, FL 33136, USA \\ wnonner@chroma.med.miami.edu \\ ROBERT S. EISENBERG ${ }^{\ddagger}$ \\ Department of Molecular Biophysics and Physiology, Rush Medical Center, Chicago, IL 60612, USA \\ beisenbe@rush.edu
}

\begin{abstract}
The drift-diffusion (Poisson-Nernst-Planck) model is applied to ionic channels in biological membranes plus surrounding solution baths. Simulations of the $\mathrm{K}$ channel in $\mathrm{KCl}$ solutions using the TRBDF2 method are presented which show significant boundary layers at the ends of the channel. The computed current-voltage curve for the $\mathrm{K}$ channel shows excellent agreement with experimental measurements.
\end{abstract}

Keywords: biological channels, diffusion models, ion transport

\section{Introduction}

Biological cells exchange chemicals and electric charge with their environments through ionic channels - protein tunnels filled with ions and waterin the cell membrane walls (see Ref. [1] for a comprehensive introduction). Signaling in the nervous system, coordination of muscle contraction including the pumping action of the heart, and ion transport in every cell and organ are carried out through ionic channels.

We consider a flow of positive and negative ions (cations and anions) in water in a channel (or pore) plus surrounding baths in an electric field $\mathbf{E}(\mathbf{x}, t)$ against a background of charged atoms on the channel protein. The discrete distribution of charges is described by con-

* Research supported in part by DARPA under grant BAA 01-07. ${ }^{\dagger}$ Research supported in part by NIH/NSF under grant R01 GM 67241-01.

${ }^{\ddagger}$ Research supported in part by DARPA under grant BAA 01-07. tinuum [2-4] particle densities $n_{i}(\mathbf{x}, t)$ for the mobile ions $\left(i=\mathrm{K}^{+}, \mathrm{Cl}^{-}, \mathrm{Na}^{+}, \mathrm{Ca}^{++}, \ldots\right)$ and $N(\mathbf{x})$ for the charged atoms ("doping" in the language of semiconductor device physics) of the protein. The permanent fixed charge density $N$ may include both positive and negative charges, but the protein is predominantly negatively charged. Note that $N$ vanishes by definition in the baths.

In the experimental setup, a voltage bias $V$ is applied across the channel plus baths by means of a voltage clamp. The channel transit time for an ion is on the order of $10 \mathrm{~ns}$, while experimentally the finest temporal resolution of currents is on the order of 10-50 $\mu \mathrm{s}$, so experimental measurements are averaging over 10005000 ions.

Although in this investigation we focus on the steady-state flow of ions, we solve the time-dependent drift-diffusion equations because we are ultimately interested in the dynamics of ion transport (especially in gating) as well, and because the computational time 
for simulating the time-dependent equations to steady state is comparable to or faster than that of steady-state solvers, which must employ a linearization scheme like Newton's method.

\section{Time-Dependent Drift-Diffusion Model}

Mathematically the flow of ions can be approximated by the drift-diffusion or Poisson-Nernst-Planck (PNP) model, that is, by partial differential equations for conservation of each ion species and Poisson's equation for the electrostatic potential $\phi(\mathbf{x}, t)$ :

$$
\begin{gathered}
\frac{\partial n_{i}}{\partial t}+\nabla \cdot \mathbf{j}_{i}=0 \\
\mathbf{j}_{i}=z_{i} \mu_{i} n_{i} \mathbf{E}-D_{i} \nabla n_{i} \\
\nabla \cdot(\epsilon \nabla \phi)=e N-\sum_{i} q_{i} n_{i}, \quad \mathbf{E}=-\nabla \phi
\end{gathered}
$$

where $e$ is the proton charge, $\epsilon$ is the dielectric coefficient, and where for each ion species $i, \mathbf{j}_{i}$ is the number current density (particle flux), $q_{i}$ is the ionic charge (for example, $\left.q_{C a}=+2 e\right), z_{i}=q_{i} / e, \mu_{i}$ is the mobility coefficient, and $D_{i}$ is the diffusion coefficient. The total electric current density (charge flux) is

$$
\mathbf{j}_{\text {elec }}=\sum_{i} q_{i} \mathbf{j}_{i}
$$

The physical parameters $\epsilon, \mu_{i}$, and $D_{i}$ are functions of $\mathbf{x}$.

The drift-diffusion equations form a parabolic/ elliptic system of PDEs: the transport equation (1) (with $\mathbf{j}_{i}$ specified by Eq. (2)) is parabolic and Poisson's equation (3) is elliptic. Thus the boundary conditions for both $n_{i}$ and $\phi$ are Dirichlet and/or Neumann.

As an example of a physiologically important channel, we will focus our attention here on the K channel illustrated in Fig. 1 (the KcsA channel structure shown is derived from X-ray crystallography). K channels play a central role in electrical signaling in the nervous system. A typical nerve cell has hundreds of thousands of $\mathrm{K}$ channels.

The $\mathrm{K}$ channel is selective; i.e., it allows $\mathrm{K}^{+}$ions to flow freely between the interior and exterior of the cell, but not (for example) $\mathrm{Na}^{+}$or $\mathrm{Ca}^{++}$ions. $\left(\mathrm{Cl}^{-}\right.$ions are prevented from flowing through the $\mathrm{K}$ channel by the electrostatic field in the channel.) We will model the channel plus regions of the bath illustrated in Fig. 2 out to a distance where the ion densities and the electrostatic potential take on their asymptotic values in

\section{Schematic view of $K$ channel structure}

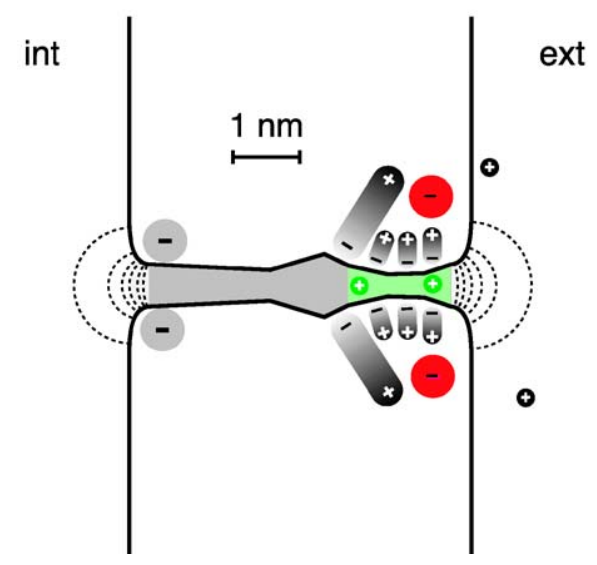

Figure 1. K channel, membrane, and interior and exterior baths.

the baths: $n_{i}=N_{b i}, \phi=0$ at the left boundary, and $n_{i}=N_{b i}, \phi=V$ at the right boundary (see Figs. 4 and 5). The overall region is electrically neutral. The diffusion and mobility coefficients of the mobile ions are typically much smaller in the channel than in the baths (see Table 1).

Boundary conditions should not be applied at the ends of the channel, due to the fact that boundary layers in ionic charge density and in the electrostatic potential develop there, as illustrated in Figs. 4 and 5. The ionic charge densities and the electrostatic potential reach their equilibrium far-field values approximately two Debye lengths into the baths (the Debye lengths for $\mathrm{K}^{+}$and $\mathrm{Cl}^{-}$are on the order of $1 \mathrm{~nm}$ in the baths). In the one-dimensional approximation to the $\mathrm{K}$ channel problem with the channel $3.5 \mathrm{~nm}$ long, the baths are represented by conical funnels (Fig. 3) extending 5 $\mathrm{nm}$ into the baths and opening at $45^{\circ}$ angles on either side of the $z$ axis (the opening angle has only a weak effect on the computed solution and total current). The funnels should be wide enough so that the impedance of the baths to the ion flow is very small compared to the impedance of the channel.

The drift-diffusion equations (1)-(3) can be written in the form

$$
\begin{gathered}
\frac{\partial n_{i}}{\partial t}+\nabla \cdot\left(z_{i} \mu_{i} \mathbf{E} n_{i}\right)=\nabla \cdot\left(D_{i} \nabla n_{i}\right) \\
\mathbf{j}_{\text {elec }}=\sum_{i} q_{i}\left(z_{i} \mu_{i} n_{i} \mathbf{E}-D_{i} \nabla n_{i}\right) \\
\nabla \cdot(\epsilon \nabla \phi)=e N-\sum_{i} q_{i} n_{i}, \quad \mathbf{E}=-\nabla \phi .
\end{gathered}
$$




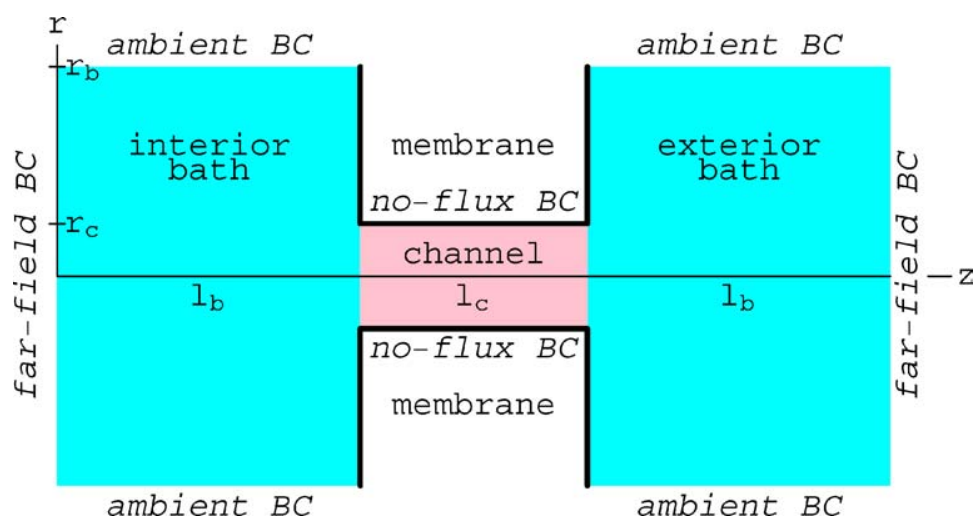

Figure 2. Diagram of computational region for channel, membrane, and baths. Boundary condition types are labeled in italics for the 3D problem.

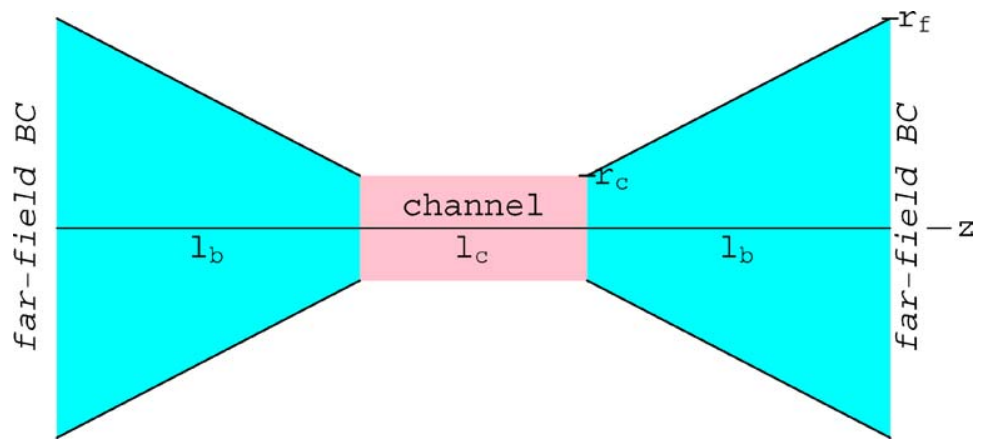

Figure 3. Diagram of 1D computational region for channel and bath funnels.

The boundary condition (BC) types in Fig. 2 are defined by

$$
\begin{aligned}
n_{i} & =N_{b i}, \quad \phi=0 & & \text { (interior bath far-field BC) } \\
n_{i} & =N_{b i}, \quad \phi=V \quad & & (\text { exterior bath far-field BC) } \\
n_{i} & =N_{b i}, \quad \frac{\partial \phi}{\partial r}=0 & & (\text { ambient bath BC) } \\
\hat{\mathbf{n}} \cdot \nabla n_{i} & =0, \quad \hat{\mathbf{n}} \cdot \nabla \phi=0 & & \text { (no-flux BC) }
\end{aligned}
$$

where $\hat{\mathbf{n}}$ is a unit normal vector to the boundary.

The channel problem illustrated in Fig. 1 has an approximate cylindrical symmetry; in other words, the solution $n_{i}, \phi$ depends to an excellent approximation only on $r$ and $z$, where cylindrical coordinates are denoted by $(r, \theta, z)$, with the $z$ axis along the length of the channel. (With cylindrical symmetry, no-flux boundary conditions are imposed along the $z$ axis as well.) If we neglect the dependence of $n_{i}$ and $\phi$ on $r$ in the channel and the near bath regions, which is a good approximation if the cross sectional area $A(z)$ varies slowly with $z$ (this is the standard approximation used in the anal- ysis and simulation of nozzle flow in gas dynamics), then we obtain a one-dimensional approximation to the channel problem.

In our first model, we will treat just a onedimensional approximation of the channel plus funnels opening into the baths on either side. Future simulations will extend the simulations to two and three dimensions.

To write down the $1 \mathrm{D}$ equations, recall that the definition of the divergence of a vector field $\mathbf{u}$ is

$$
\nabla \cdot \mathbf{u}=\lim _{\mathcal{V} \rightarrow 0} \frac{1}{\mathcal{V}} \int_{\partial \mathcal{V}} \mathbf{u} \cdot \hat{\mathbf{n}} d a
$$

where $\mathcal{V}$ is a volume centered around $\mathbf{x}$ with bounding surface $\partial \mathcal{V}$. For $\mathbf{u}(\mathbf{x})=u(z) \hat{\mathbf{e}}_{z}$ and $\mathcal{V}=A(z) \Delta z$, Eq. (8) reduces to

$$
\begin{aligned}
\nabla \cdot \mathbf{u} & =\lim _{\Delta z \rightarrow 0} \frac{1}{A \Delta z}[u(z+\Delta z) A(z+\Delta z)-u(z) A(z)] \\
& =\frac{1}{A} \frac{\partial}{\partial z}(A u)
\end{aligned}
$$


by Taylor expanding in $\Delta z$. (Note that the gradient is unchanged: $\nabla \phi=\partial \phi / \partial z$ for $\phi(\mathbf{x})=\phi(z)$.)

The drift-diffusion equations thus become in the $1 \mathrm{D}$ approximation

$$
\begin{gathered}
\frac{\partial n_{i}}{\partial t}+\frac{1}{A} \frac{\partial}{\partial z}\left(A z_{i} \mu_{i} E n_{i}\right)=\frac{1}{A} \frac{\partial}{\partial z}\left(A D_{i} \frac{\partial n_{i}}{\partial z}\right), \\
n_{i}(0, t)=N_{b i}=n_{i}(L, t) \\
j_{\text {elec }}=\sum_{i} q_{i}\left(z_{i} \mu_{i} n_{i} E-D_{i} \frac{\partial n_{i}}{\partial z}\right) \\
\frac{1}{A} \frac{\partial}{\partial z}\left(\epsilon A \frac{\partial \phi}{\partial z}\right)=e N-\sum_{i} q_{i} n_{i}, \quad E=-\frac{\partial \phi}{\partial z}, \\
\phi(0)=0, \quad \phi(L)=V
\end{gathered}
$$

where the simulation region which includes the channel plus baths runs from 0 to $L=l_{c}+2 l_{b}$ and $n_{i}(\mathbf{x}, t)=n_{i}(z, t), \phi(\mathbf{x}, t)=\phi(z, t)$. For both the elliptic Poisson's equation (12) and the parabolic transport equation (10), Dirichlet boundary conditions on $\phi$ and $n_{i}$ are imposed at the left and right boundaries.

Note that in this 1D approximation, the current density (and thus the ion velocity) and the electric field will lie in the $z$ direction. To simplify the mathematical analysis of the 1D model (10)-(12), the cross sectional area $A(z)$ may be set to a constant in the channel itself, but not in the baths.

The drift-diffusion model for a spherically symmetric problem $n_{i}(\mathbf{x}, t)=n_{i}(r, t), \phi(\mathbf{x}, t)=\phi(r, t)$, where spherical coordinates are denoted by $(r, \theta, \varphi)$, takes exactly the same form as Eqs. (10)-(12) with $z$ replaced by $r$. Since the equipotential surfaces and the level set curves of $n_{i}$ are normal to the cell membrane walls (since there is no ion flow through the walls and since to a good approximation there is no electrical flux through the walls), the solution in the baths near the channel openings may be modeled as approximately spherically symmetric [5] for a moderate radial distance into the baths. Then, as long as the flow and electric field lines remain approximately radial in the baths near the channel openings, Eqs. (10)-(12) with $z$ replaced by $r$ may be used with the cross sectional area $A(r)$ now the surface area of spherical shells starting and ending at a cell membrane wall. The $1 \mathrm{D}$ cylindrically symmetric solution in the channel is "patched" onto the spherically symmetric solution in the channel opening and the bath. Both the assumption of spherical symmetry and the patching together of solutions introduce errors. The validity of this approximation will be tested against 2D cylindrically symmetric simulations.

\section{Steady-State Drift-Diffusion Model}

Although we will solve the time-dependent driftdiffusion equations below, for completeness we also give the steady-state equations and associated boundary conditions.

For the flow of cations and anions in steady state, the drift-diffusion equations (1)-(3) take the form $\nabla \cdot \mathbf{j}_{i}=$ 0 , or in other words

$$
\begin{gathered}
\nabla \cdot\left(z_{i} \mu_{i} \mathbf{E} n_{i}-D_{i} \nabla n_{i}\right)=0 \\
\mathbf{j}_{\text {elec }}=\sum_{i} q_{i}\left(z_{i} \mu_{i} n_{i} \mathbf{E}-D_{i} \nabla n_{i}\right) \\
\nabla \cdot(\epsilon \nabla \phi)=e N-\sum_{i} q_{i} n_{i}, \quad \mathbf{E}=-\nabla \phi .
\end{gathered}
$$

In $1 \mathrm{D}$, the steady-state drift-diffusion equations are

$$
\begin{gathered}
\frac{\partial}{\partial z}\left(A\left(z_{i} \mu_{i} E n_{i}-D_{i} \frac{\partial n_{i}}{\partial z}\right)\right)=0, \\
n_{i}(0)=N_{b i}=n_{i}(L) \\
j_{\text {elec }}=\sum_{i} q_{i}\left(z_{i} \mu_{i} n_{i} E-D_{i} \frac{\partial n_{i}}{\partial z}\right) \\
\frac{1}{A} \frac{\partial}{\partial z}\left(\epsilon A \frac{\partial \phi}{\partial z}\right)=e N-\sum_{i} q_{i} n_{i}, \quad E=-\frac{\partial \phi}{\partial z}, \\
\phi(0)=0, \phi(L)=V .
\end{gathered}
$$

\section{Numerical Methods for Electrodiffusion}

Variables $n_{i}$ and $\phi$ are defined at gridpoints $0,1, \ldots, N$, while $j$ and $E$ are defined at midpoints of grid cells $-1 / 2,1 / 2,3 / 2, \ldots, N+1 / 2$. Given $n_{i}^{n}$ and $E^{n}$ at timelevel $n$, a timestep consists of two parts. (i) First we solve the transport equation (10) for $n_{i}^{n+1}$ with $E=E^{n}$. (ii) Then we solve Poisson's equation (12) for $E^{n+1}$ using $n_{i}^{n+1}$ on the right-hand side.

We use the implicit, L-stable TRBDF2 [6,7] (trapezoidal rule/second-order backward difference formula) method for the time-dependent drift-diffusion transport equation, which allows simulations to use large timesteps. TRBDF2 is a one-step second-order accurate method; the timestep is adjusted dynamically by a divided difference formula estimate of the local error.

After discretizing in space, the transport equation (10) may be written as a system of ordinary differential equations $d u / d t=f(u)$. The TRBDF2 method 
then takes the form-(1) TR step of $\gamma \Delta t$ :

$$
u^{n+\gamma}-\gamma \frac{\Delta t_{n}}{2} f^{n+\gamma}=u^{n}+\gamma \frac{\Delta t_{n}}{2} f^{n}
$$

and (2) BDF2 step of $\Delta t$ :

$$
\begin{aligned}
& u^{n+1}-\frac{1-\gamma}{2-\gamma} \Delta t_{n} f^{n+1} \\
& =\frac{1}{\gamma(2-\gamma)} u^{n+\gamma}-\frac{(1-\gamma)^{2}}{\gamma(2-\gamma)} u^{n} .
\end{aligned}
$$

With the electric field frozen while updating the transport equation, $f(u)$ is linear. We take the usual value $\gamma=2-\sqrt{2}$ which minimizes the magnitude of the local error.

For Poisson's equation we use a tridiagonal direct solver.

\section{Simulation of the K Channel}

We will consider here the flow of $\mathrm{K}^{+}$ions (in water) through a channel of diameter $1 \mathrm{~nm}$ and length $3.5 \mathrm{~nm}$.

For various sections of the $\mathrm{K}$ channel and surrounding $\mathrm{KCl}$ baths, the lengths $l$, background permanent charges $Q$ on the protein which go into $N$, dielectric constants $\epsilon$, mobility coefficients $\mu$, and diffusion coefficients $D$ are given in Table 1 . The mobilities and diffusion coefficients satisfy the Einstein relation $e D / \mu=k_{B} T_{0} \approx 1 / 40 \mathrm{eV}$.

The bath concentrations for the positive and negative ions are 0.15 molar $=9 \times 10^{19} \mathrm{~cm}^{-3}$. We also
Table 1. Lengths $l$ in nm, background permanent charges $Q$ on the protein, dielectric constants $\epsilon$, mobility coefficients $\mu$ in $10^{-5} \mathrm{~cm}^{2} /(\mathrm{V} \mathrm{s})$, and diffusion coefficients $D$ in $10^{-5} \mathrm{~cm}^{2} / \mathrm{s}$.

\begin{tabular}{llcccc}
\hline Region & $l$ & $Q$ & $\epsilon$ & $\mu$ & $D$ \\
\hline Baths & 5 & 0 & 80 & 60 & 1.5 \\
$-4 e$ group & 0.2 & $-4 e$ & 80 & 16 & 0.4 \\
Nonpolar & 1.1 & 0 & 4 & 16 & 0.4 \\
Central cavity & 1 & $-e / 2$ & 30 & 16 & 0.4 \\
Filter & 1.2 & $-3 e / 2$ & 30 & 16 & 0.4 \\
\hline
\end{tabular}

assume that there are equal concentrations of ions inside and outside the cell membrane, so that no current flows when $V=0$. The baths are modeled by funnels which open from the channel cross sectional area $A=\pi(1 \mathrm{~nm} / 2)^{2}$ at the channel opening out to $A=\pi(11 \mathrm{~nm} / 2)^{2}$ at $5 \mathrm{~nm}$ into the baths. These parameters give a linear current-voltage curve with a current of 22.5 picoamperes at a bias of -100 millivolts (Fig. 6). Experimentally the current at -100 millivolts is on the order of 20-30 picoamps for this type of channel.

The computed ion densities and electrostatic potential are shown in Figs. 4 and 5: $z$ is in $n$, and the channel lies between $z=0$ and $z=3.5$. The baths extend $5 \mathrm{~nm}$ on either side of the channel (only part of the baths is shown in Fig. 4). The ion flow is from the interior (left) to the exterior (right) of the cell membrane. The total charge on the channel is $-6 e$; there are 4.3 $\mathrm{K}^{+}$ions on average flowing in the channel, with about two $\mathrm{K}^{+}$ions on average in the neighborhood of the

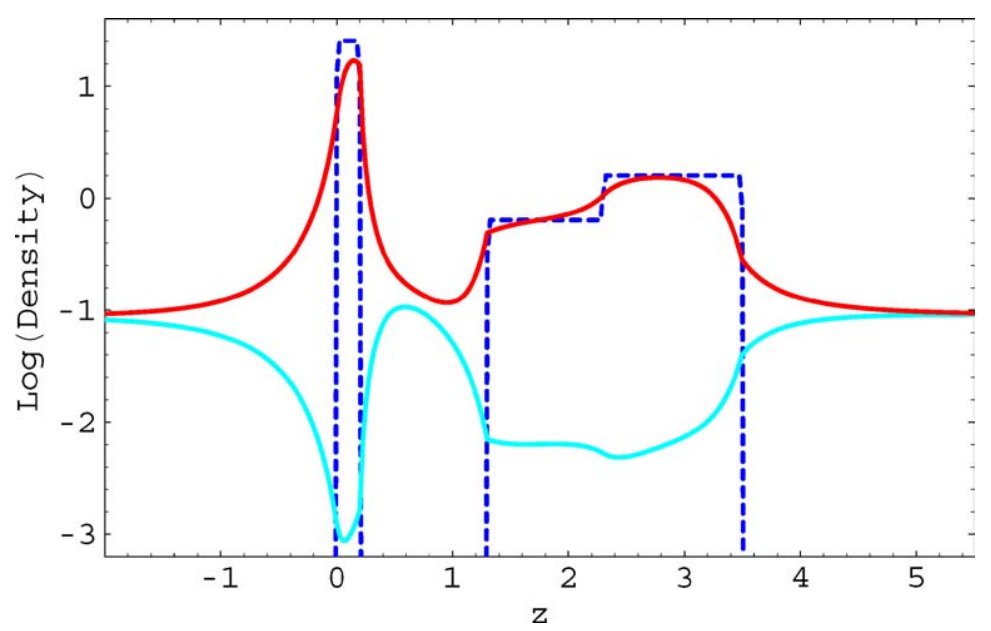

Figure 4. Simulation of the $\mathrm{K}^{+}$density $n_{K}$ (red, dark) and $\mathrm{Cl}^{-}$density $n_{\mathrm{Cl}}$ (cyan, light) for $V=-100$ millivolts. The protein permanent charge density $N$ is shown in blue (dashed). The vertical scale is $\log _{10}$ of density/ $\left(10^{21} \mathrm{~cm}^{-3}\right)$. 


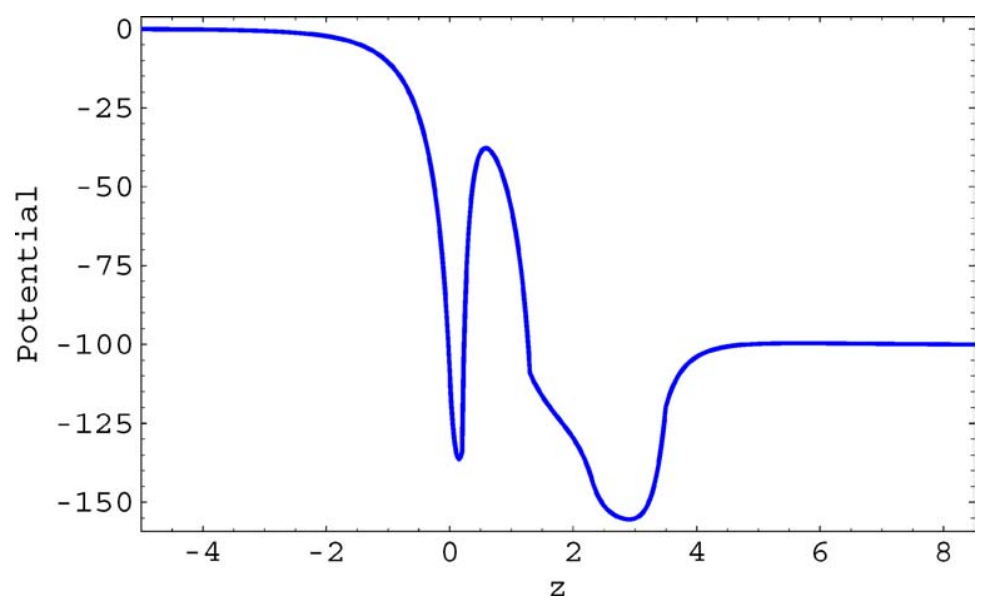

Figure 5. Simulation of electrostatic potential in millivolts for $V=-100$ millivolts.

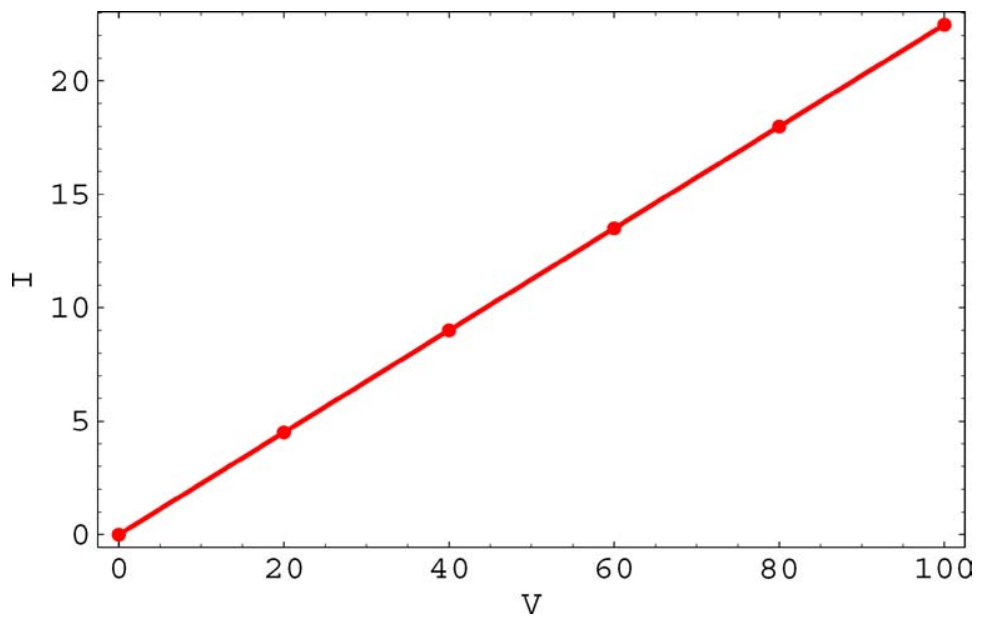

Figure 6. Current in picoamperes vs. voltage in millivolts.

$-4 e$ charge group and about one $\mathrm{K}^{+}$ion on average in the filter. (The drift-diffusion model predicts about $1 / 15 \mathrm{Cl}^{-}$ion on average flowing in the channel; this is a reasonable continuum approximation to the actual biological picture of discrete ions in which $\mathrm{Cl}^{-}$ions are excluded by the electrostatic field from the $\mathrm{K}$ channel.) The simulated region of channel plus baths is electrically neutral overall.

At $V=-100$ millivolts, the current $I_{+}$carried by the $\mathrm{K}^{+}$ions is 22.2 picoamps, while the current $I_{-}$ carried by the $\mathrm{Cl}^{-}$ions is 0.3 picoamps. Many experimentalists believe that no current should be carried by the $\mathrm{Cl}^{-}$ions; however measurements only restrict $I_{-}<I / 20$.

\section{Conclusion}

The fact that the simulations of the $\mathrm{K}^{+}$and $\mathrm{Cl}^{-}$ion densities and the current-voltage curve agree well with experimentally measured or derived properties of the $\mathrm{K}$ channel gives credence to the continuum fluid approximation to the ion flow. For $V=-100$ millivolts, the simulations predict about $4.5 \mathrm{~K}^{+}$ions in the channel, which is consistent with the charge structure of the protein known from X-ray crystallography. In addition, the boundary layers in the ion densities and electrostatic potential extend roughly two Debye lengths into the baths, and the maximum magnitude of the electrostatic potential is safely below the biologically dangerous 
value of 200 millivolts at which the lipid membrane begins to break down from charge arcing and the channel protein itself is deformed by the electrostatic forces.

Future work will include simulations for the 2D cylindrically symmetric approximation to the channel plus baths, and a separate model for gating and gating charge movement.

\section{References}

1. B. Hille, Ionic Channels of Excitable Membranes (Sinauer, Sunderland, MA, 1992).

2. R.S. Eisenberg, M.M. Klosek, and Z. Schuss, "Diffusion as a chemical reaction: Stochastic trajectories between fixed concentrations," Journal of Chemical Physics, 102, 1767 (1995).
3. W. Nonner, D. Chen, and R.S. Eisenberg, "Anomalous mole fraction effect, electrostatics, and binding," Biophysical Journal, 74, 2327 (1998).

4. W. Nonner and R.S. Eisenberg, "Ion permeation and glutamate residues linked by Poisson-Nernst-Planck theory in L-type calcium channels," Biophysical Journal, 75, 1287 (1998).

5. D. Gillespie, W. Nonner, and R.S. Eisenberg, "Coupling PoissonNernst-Planck and density functional theory to calculate ion flux," Journal of Physics: Condensed Matter, 14, 12129 (2002).

6. R.E. Bank, W.M. Coughran, W. Fichtner, E.H. Grosse, D.J. Rose, and R.K. Smith, "Transient simulation of silicon devices and circuits," IEEE Transactions on Computer-Aided Design, 4, 436 (1985).

7. R.B. Fair, C.L. Gardner, M.J. Johnson, S.W. Kenkel, D.J. Rose, J.E. Rose, and R. Subrahmanyan, "Two dimensional process simulation using verified phenomenological models," IEEE Transactions on Computer-Aided Design of Integrated Circuits and Systems, 10, 643 (1991). 